\title{
Improving Patient-Centered Communication about Sudden Unexpected Death in Epilepsy through Computerized Clinical Decision Support
}

\author{
Randall W. Grout ${ }^{1,2}$ Jeffrey Buchhalter ${ }^{3}$ Anup D. Patel ${ }^{4}$ Amy Brin ${ }^{5}$ Ann A. Clark ${ }^{1}$ Mary Holmay ${ }^{6}$ \\ Tyler J. Story ${ }^{6,7}$ Stephen M. Downs ${ }^{1,2}$
}

${ }^{1}$ Department of Pediatrics, Children's Health Services Research, Indiana University, Indianapolis, Indiana, United States

${ }^{2}$ Center for Biomedical Informatics, Regenstrief Institute, Inc., Indianapolis, Indiana, United States

${ }^{3}$ Department of Pediatrics, University of Calgary, Section of Neurology, Alberta Children's Hospital, Calgary, Canada

${ }^{4}$ Division of Neurology, Nationwide Children's Hospital and Department of Pediatrics, The Ohio State University College of Medicine, Columbus, Ohio, United States

${ }^{5}$ Child Neurology Foundation, Minneapolis, Minnesota, United States

${ }^{6}$ Greenwich Biosciences, Carlsbad, California, United States (at the time of this study)

${ }^{7}$ UCB, Inc., Smyrna, Georgia, United States

Appl Clin Inform 2021;12:90-99.

\section{Abstract}

Keywords

- clinical decision support systems

- sudden unexpected death in epilepsy

- risk factors

- patient education

- communication
Address for correspondence Randall W. Grout, MD, MS, Department of Pediatrics, Children's Health Services Research, Indiana University, 410 West 10th Street, HS 2000, Indianapolis, IN 46202, United States (e-mail: rgrout@iu.edu). received

July 8,2020

accepted after revision

November 18, 2020
Background Sudden unexpected death in epilepsy (SUDEP) is a rare but fatal risk that patients, parents, and professional societies clearly recommend discussing with patients and families. However, this conversation does not routinely happen.

Objectives This pilot study aimed to demonstrate whether computerized decision support could increase patient communication about SUDEP.

Methods A prospective before-and-after study of the effect of computerized decision support on delivery of SUDEP counseling. The intervention was a screening, alerting, education, and follow-up SUDEP module for an existing computerized decision support system (the Child Health Improvement through Computer Automation [CHICA]) in five urban pediatric primary care clinics. Families of children with epilepsy were contacted by telephone before and after implementation to assess if the clinician discussed SUDEP at their respective encounters.

Results The CHICA-SUDEP module screened 7,154 children age 0 to 21 years for seizures over 7 months; 108 (1.5\%) reported epilepsy. We interviewed 101 families after primary care encounters ( 75 before and 26 after implementation) over 9 months. After starting CHICA-SUDEP, the number of caregivers who reported discussing SUDEP with their child's clinician more than doubled from $21 \%(16 / 75)$ to $46 \%$ (12/26; $p=0.03$ ), and when the parent recalled who brought up the topic, $80 \%$ of the time it was the clinician. The differences between timing and sampling methodologies of before and after intervention cohorts could have led to potential sampling and recall bias. 
Conclusion Clinician-family discussions about SUDEP significantly increased in pediatric primary care clinics after introducing a systematic, computerized screening and decision support module. These tools demonstrate potential for increasing patientcentered education about SUDEP, as well as incorporating other guideline-recommended algorithms into primary and subspecialty cares.

Clinical Trial Registration clinicaltrials.gov, NCT03502759.

\section{Background and Significance}

Children with epilepsy have an estimated risk of sudden unexpected death in epilepsy (SUDEP) between 1 in 1,000 and 1 in 4,500 per year., ${ }^{1,2}$ For that reason, the American Academy of Neurology and the American Epilepsy Society recommend that clinicians caring for these children make their families aware of this rare but fatal risk and provide appropriate supportive follow-up resources. ${ }^{3}$ SUDEP risk can increase with several factors including lack of nocturnal supervision and some genetic causes of epilepsy (e.g., Dravet's syndrome). ${ }^{4}$ Moreover, existing evidence suggests that children with generalized tonic-clonic seizures (GTCs) have strikingly increased odds of SUDEP, 5 to 15 folds, raising the importance of improving seizure control. ${ }^{3}$

Clinicians caring for these patients have multiple issues to address in the typical visit. We developed and evaluated health information technology to help providers incorporate SUDEP counseling into routine care. We previously developed the Child Health Improvement through Computer Automation system (CHICA), a computer-based clinical decision support system (CDSS) for pediatric care. ${ }^{5}$ CHICA captures patient-reported data in the waiting room and prioritizes clinical advice to the clinician through the electronic health record (EHR).

\section{Objectives}

The goal of this pilot study was to test the effectiveness of a CHICA-SUDEP module to improve clinician-patient communication about SUDEP, including help overcoming barriers to seizure control, in a setting where CHICA is already in use. Additionally, we sought to understand patient educational experiences and knowledge about SUDEP generally to later inform approaches to education.

\section{Methods}

\section{Evaluation of the CHICA-SUDEP Module}

We evaluated clinical decision support (the CHICA-SUDEP module) in a before-after study design in five primary care clinics of Eskenazi Health, a large, urban safety-net health care system in Indianapolis, Indiana, United States. To determine if the module increased discussions of SUDEP, we conducted a telephone survey of parents or caregivers of children with epilepsy before and after the CHICA-SUDEP module was added to an existing decision support system (CHICA) in the five primary care clinics that use it at Eskenazi
Health ("CHICA clinics"). The study was approved by the Indiana University Institutional Review Board (IRB).

\section{Child Health Improvement through Computer Automation}

Investigators at Indiana University School of Medicine developed a software system that offers an innovative solution to the high demands of caring for children. CHICA is a CDSS that works with a provider's EHR to capture data directly from patients, deliver alerts and reminders to clinicians, and quickly document services provided. CHICA has been the subject of dozens of clinical studies that consistently show improved adherence to clinical guidelines. ${ }^{5-9}$

CHICA has been described in detail elsewhere. ${ }^{5}$ Briefly, CHICA uses industry-standard Arden Syntax decision rules ${ }^{10}$ and the Health Level Seven (HL7) standard for information exchange for ease of implementation in EHR systems. At each visit, the EHR registration system sends a registration message with portions of the patient's record to CHICA to trigger its decision support. CHICA applies hundreds of rules to the data to select the 20 highest priority questions. The parent/guardian receives a tablet at check-in with the 20 questions, displayed five at a time on four sequential screens. The questions are produced in both English and Spanish, and the parent/guardian can toggle between them.

When the parent/guardian's answers are submitted, an agenda for the clinician is generated in an analogous process. CHICA applies hundreds of other rules to the child's record and the family's answers to select six prompts (i.e., structured information with recommendations) to show the clinician (-Fig. 1). The clinician accesses these alerts from within the EHR. Each clinician alert includes a "stem" that explains the purpose of the alert. The clinician responds by checking boxes that document his or her response, which are saved for future use. CHICA also produces patient handouts, like smoking cessation advice and developmental screening instruments, tailored to each patient. A summary of the patient/caregiver and clinician interactions with CHICA are written as prose into the clinician's note in the EHR.

\section{The CHICA-SUDEP Module}

CHICA can be adapted to incorporate clinical guidelines into a "module" by mapping those guidelines into a set of rules (medical logic modules [MLMs]) coded into Arden Syntax. The MLMs control which questions are asked to families, which alerts are given to clinicians, and which patient educational materials and other clinical tools are made available at the encounter. 


\section{Ima has had 5 seizures in the past year, putting her at INCREASED RISK for sudden death (SUDEP). Family reports: Missed meds = more than 5, parent sometimes forgets child's meds, child hasn't seen a neurologist in over a year, and it is recommended that you discuss SUDEP (Handout).}

\section{$\checkmark$ Discuss risk of SUDEP Referred to Neurol No Seizures past 12 mos}

\author{
$\checkmark$ Shared handout \\ Discussed meds \\ Does NOT have epilepsy
}

Fig. 1 Screenshot of clinician prompt. In this example, the family has reported multiple issues with seizures, medications and seeing the neurologist. The clinician has documented a response. SUDEP, sudden unexpected death in epilepsy.

The intervention in this study was the SUDEP module. The SUDEP module was based on recommendations from the American Academy of Neurology (AAN). ${ }^{3}$ A project team of informaticians, child neurologists, pediatricians, health services researchers, and parents of children with epilepsy was convened by the Child Neurology Foundation to develop a clinical algorithm to define the function of the CHICASUDEP module. This process started with a "strawman algorithm" developed by the lead informatician (S.M.D.). In a modified nominal group process, the team modified the algorithm until consensus was reached (-Fig. 2).

The module was triggered for any child for whom a diagnosis of epilepsy was not known based on data collected through CHICA. It asked the family if the child had epilepsy or more than one seizure with stiffness or jerking, to identify children with GTCs. ${ }^{11,12}$ If the answer was "yes," CHICA would display a series of follow-up questions, assessing the number of seizures in the last year, the medication adherence, whether the child had seen a neurologist, and barriers to medication adherence or seeing the neurologist. Questions that could help the physician identify barriers to epilepsy treatment were derived from the Pediatric Epilepsy Medication Self-Management Questionnaire (PEMSQ; see appendix for questions used in this module [-Supplementary Appendix A, available in the online version]). ${ }^{13}$ Although the SUDEP guidelines recommend SUDEP education for all epilepsy patients, the intention of this pilot study was to identify patients with a higher risk (those having GTC seizures) compared with those that were not. Therefore, the education was differentiated as higher risk compared with lower risk based on our assessment of the presence of GTC seizures.

We recognize that seizures alone are not the risk factor of interest for SUDEP. Our screening question is similar to that of prior studies that show it to be a reasonable surrogate for identifying GTCs. ${ }^{11,12}$ Therefore, we refer to these patients as having GTCs, while acknowledging the risk of misclassifying children with other seizure types that may not be a driver of increased SUDEP risk.

If the family had reported epilepsy or GTC seizures, when the clinician opened the EHR and accessed the CHICA agenda, one of the prompts on the agenda would alert the clinician that the child had epilepsy or GTC seizures, as well as any adherence problems or barriers to care that the parent reported on the follow-up questionnaire. If the parent reported one or more GTC seizures in the preceding year, CHICA was programmed to indicate that the child was at increased risk for $\operatorname{SUDEP}(-$ Fig. 1 ) and generate a "high-risk SUDEP" handout for the clinician to share with the family. The intention of high-risk identification, counseling, and follow-up actions was to reduce the seizures to as few as possible. Otherwise, the prompt and handout indicated low risk for SUDEP. The clinicians could respond to the alert by indicating that SUDEP counseling was done, they shared the handout, they referred to neurology, they discussed medications, or that the child did not have epilepsy. If a physician had discussed SUDEP before, they could check "discuss risk of SUDEP." Counseling and the handout for lowrisk patients communicated that the current risk was low but awareness was still important. Regardless of identified risk, both the high-risk and low-risk counseling handouts were always available to the clinician.

The "high-risk" and "low-risk" handouts were developed by the multidisciplinary team which included advocacy and caregiver stakeholders in collaboration with the Child Neurology Foundation (freely available online). ${ }^{14}$ The family questionnaires and the handouts were all generated in both English and Spanish (translated by a third-party professional service). Before the SUDEP module was launched, we sent an e-mail to the clinicians and staff who use CHICA and two authors (R.W.G. and S.M.D.) met with each of the clinics to alert them to the module and explain the importance of discussing SUDEP with families of children with epilepsy.

\section{Participants}

Participants were families of children up to 21 years old with epilepsy seen before or after the implementation of the CHICA-SUDEP module.

Before implementing the CHICA-SUDEP module, we identified children with epilepsy by searching the EHRs of patients aged 0 to 21 years who had been seen in the previous year at one of the five CHICA clinics and who had been assigned at least 


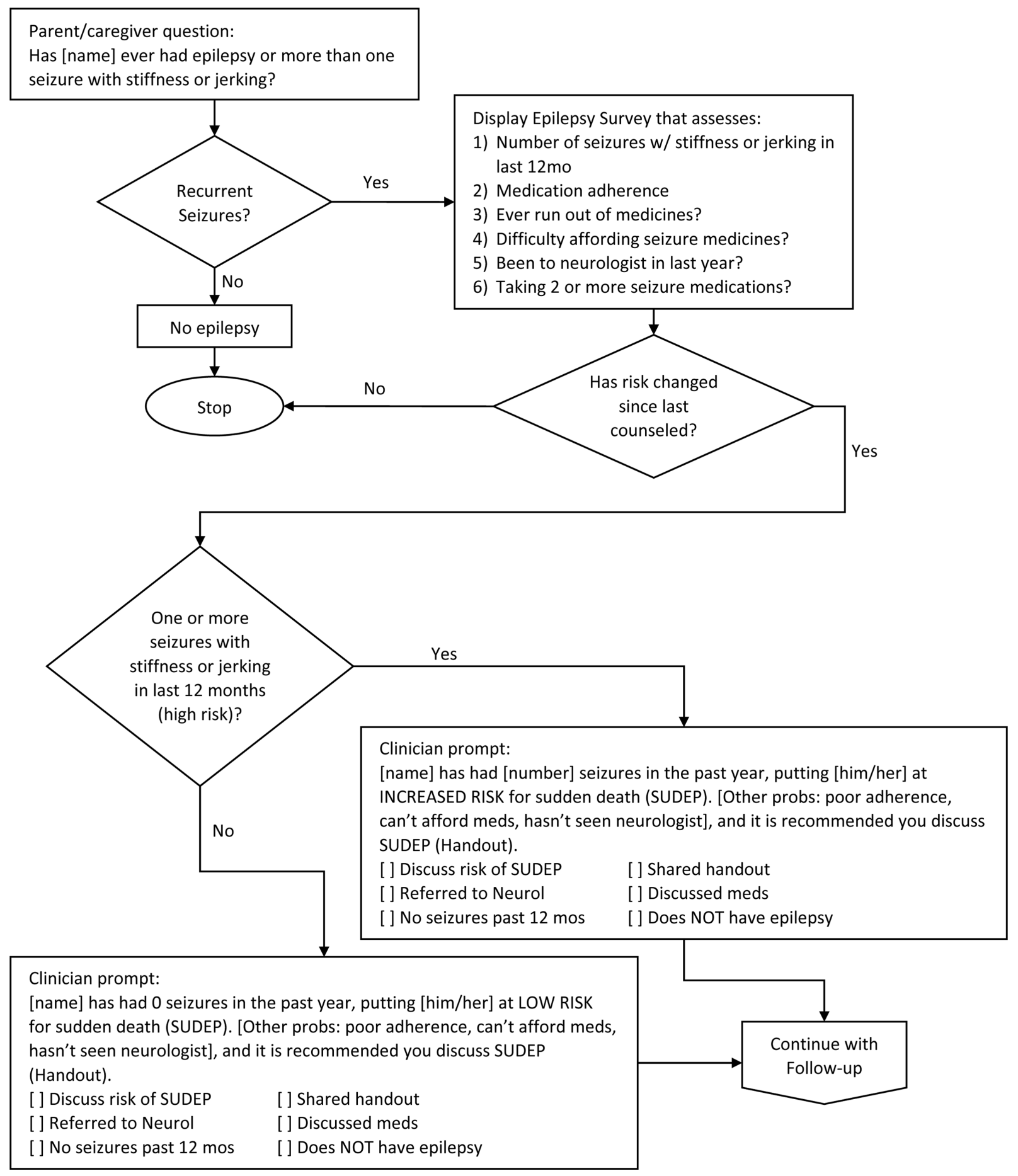

Fig. 2 A CHICA-SUDEP algorithm. CHICA, child health improvement through computer automation; SUDEP, sudden unexpected death in epilepsy.

one of the following diagnostic codes from the International Classification of Diseases-Clinical Modification (ICD-CM, versions 9 and 10) coding system: G40.* and 345.*. While these codes could include single seizures or nonepileptic events, we aimed for sensitive inclusion criteria (using wildcard code searches) with follow-up verbal confirmation. A random sample of these families was contacted for the phone survey before having a visit that used the CHICA-SUDEP module.

After implementing the CHICA-SUDEP module, we identified children whose families reported their children had epilepsy through CHICA. They were contacted by phone using the EHR-documented phone number as soon as possible after their visits. There was no overlap in the families selected for the before- and after-implementation surveys.

\section{Telephone Survey}

To determine if SUDEP had been discussed with the family, we conducted a telephone survey. Phone calls were attempted at least three times. Phone interviews were conducted by research assistants with the adult who had accompanied the 
child to the clinic visit. Respondents provided verbal informed consent, and the child's diagnosis of epilepsy was confirmed by asking, "Does (patient's name) have epilepsy or seizures with stiffness or jerking?" Any disagreement on silently coded answers (where research assistants choose from a list of discrete answers based on a narrative response from the participant) was resolved by consensus with investigators after consulting with the research assistant and reviewing their notes.

Survey content included the respondent's relationship to the child, who provided the majority of the child's epilepsy medical care, whether the parent felt he or she had a good enough understanding about epilepsy to manage it, and how much he or she worried about the risk of death related to epilepsy. We asked if the doctor talked about SUDEP at the visit to the CHICA clinic and who initiated that conversation (family or provider). Additional survey text is available in - Supplementary Appendix B (available in the online version). If a respondent was concerned or wanted more information, phone numbers and web sites to the Child Neurology Foundation and Epilepsy Foundation were provided.

\section{Analysis}

Our primary outcome was the proportion of eligible patients who had discussed SUDEP with their clinician. We used parent or caregiver report of a SUDEP discussion as the marker of a discussion, aiming for a more patient-centered outcome, and separately reported whether the clinician indicated discussing SUDEP. We compared SUDEP counseling rates before and after the intervention using a two-tailed Chi-squared statistical $(\alpha=0.05)$ analysis. The study was powered (prespecified) to detect a $30 \%$ absolute increase in counseling, assuming baseline counseling was $15 \%$ ( $\alpha=0.05$

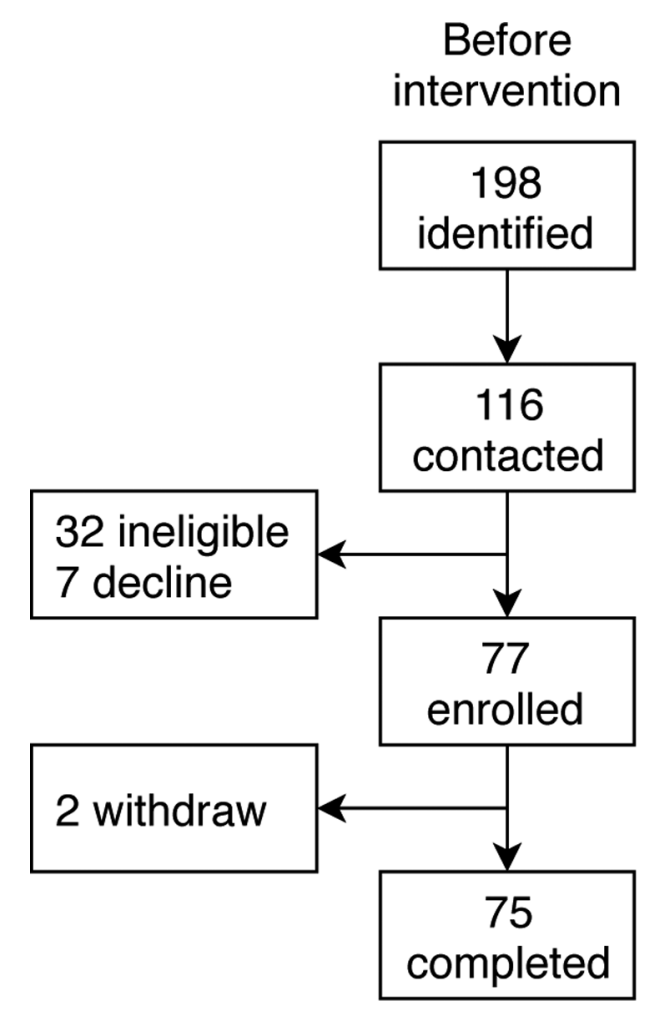

and $\beta=0.20$ ). This resulted in a sample size of 35 per group. We overrecruited to compensate for losses inherent in phone surveys. We used Microsoft Excel and R statistical software for analysis. ${ }^{15}$ Survey results were collected using Research Electronic Data Capture (REDCap). ${ }^{16}$

\section{Results}

\section{CHICA-SUDEP Implementation}

The CHICA-SUDEP module screened for seizures in 7,154 children in primary care clinics during an approximately 7-month study period postintervention. Of those, 108 (1.5\%) reported epilepsy or more than one seizure with stiffness or jerking in the past year. Ninety-one (84\%) of them reported the number of seizures, where 46 (54\%) had at least one seizure with stiffness and jerking in the preceding year. Ninety-two (85\%) completed at least part of the CHICA-SUDEP follow-up questionnaire. In 24 (22\%) of the 108 patients initially reporting epilepsy, the clinician responded to the SUDEP prompt by indicating that the child did not have epilepsy (which would suppress the module for 2 years), and in 51 (47\%), the clinician indicated that he or she discussed SUDEP.

\section{Telephone Survey Results}

Phone contact was attempted for 306 families, 198 before and 108 after. Contact was made with 201 (66\%): 116 (59\%) before and 85 (79\%) after intervention. In preintervention, 77 (92\%) of eligible participants enrolled compared with 25 (65\%) in postintervention. - Fig. 3 shows the number of respondents contacted by phone, how many were eligible and how many completed the survey. Preintervention interviews were done from June 14, 2018 until October 30, 2018, and postintervention

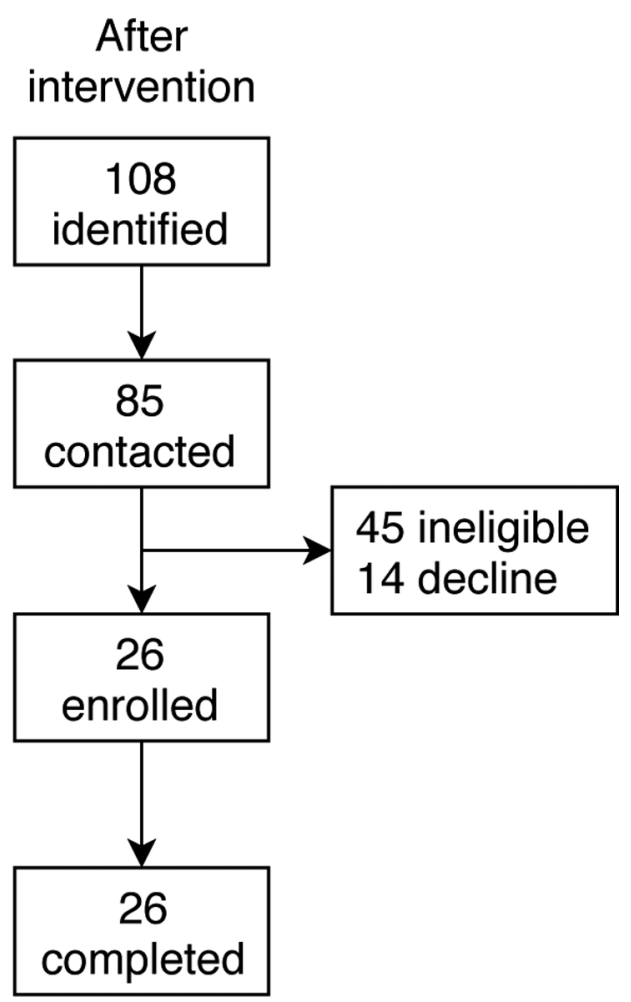

Fig. 3 Survey recruitment flowchart. 
interviews were between August 31, 2018 and March 5, 2019.In total, 101 families completed a telephone survey. The eligibility rates between the two cohorts were different, which we attribute to the difference in sampling methods, ICD diagnosis codes before the intervention and caregiver questionnaire after the intervention.

- Table 1 shows the characteristics of the children in families recruited for the study in the before- and after-intervention cohorts. Children represented both sexes. They were predominantly Black and non-Hispanic. Respondents were almost always a parent of the child. The cohorts were comparable in terms of sex, race, ethnicity, relationship of respondent to child, and child's age. The time between the index visit and the phone survey was longer in the before-intervention group (mean: 265 vs. 14 days, $p<0.001$ ) because of the difference in the way children were identified (preintervention using a one-time data review compared with postintervention in real-time as they used the module).

-Table 2 shows results of the survey before and after introduction of the CHICA-SUDEP module. Only 16 (21\%) of

Table 1 Characteristics of the recruited study populations

\begin{tabular}{|c|c|c|c|}
\hline & $\begin{array}{l}\text { Before } \\
\text { intervention } \\
n(\%)\end{array}$ & $\begin{array}{l}\text { After } \\
\text { intervention } \\
n(\%)\end{array}$ & $p$-Value ${ }^{a}$ \\
\hline Number & 75 & 26 & \\
\hline Sex (female) & $39(52)$ & $9(35)$ & 0.19 \\
\hline Race & & & 0.79 \\
\hline $\begin{array}{l}\text { American Indian/ } \\
\text { Alaskan Native }\end{array}$ & $1(1.3)$ & $0(0.0)$ & \\
\hline Black & $50(67)$ & $19(73)$ & \\
\hline Caucasian/White & $12(16)$ & $2(7.7)$ & \\
\hline $\begin{array}{l}\text { More than } \\
\text { one race }\end{array}$ & $6(8.0)$ & $3(11)$ & \\
\hline $\begin{array}{l}\text { Unknown/ } \\
\text { unreported }\end{array}$ & $6(8.0)$ & $2(7.7)$ & \\
\hline Ethnicity & & & 0.84 \\
\hline $\begin{array}{l}\text { Hispanic } \\
\text { or Latino } \\
\end{array}$ & $11(15)$ & $4(15)$ & \\
\hline $\begin{array}{l}\text { Non-Hispanic } \\
\text { or Latino }\end{array}$ & $63(84)$ & $22(85)$ & \\
\hline $\begin{array}{l}\text { Unknown/ } \\
\text { unreported }\end{array}$ & $1(1.3)$ & $0(0.0)$ & \\
\hline $\begin{array}{l}\text { Relationship } \\
\text { to patient }\end{array}$ & & & 0.80 \\
\hline Parent & $72(96)$ & $25(96)$ & \\
\hline Grandparent & $2(2.7)$ & $1(3.8)$ & \\
\hline Aunt/uncle & $1(1.3)$ & $0(0.0)$ & \\
\hline $\begin{array}{l}\text { Patient age (y) } \\
\text { Median } \\
\text { (interquartile } \\
\text { range) }\end{array}$ & $\begin{array}{l}10.2 \\
(4.3-15.4)\end{array}$ & $\begin{array}{l}8.3 \\
(3.3-11.2)\end{array}$ & 0.27 \\
\hline $\begin{array}{l}\text { Days since visit } \\
\text { Mean (SD) }\end{array}$ & 265 (107) & $14.2(21.0)$ & $<0.001$ \\
\hline
\end{tabular}

${ }^{a}$ Chi-square analysis for categorical variables, $t$-test for continuous variables. families in preintervention interviews reported that their clinician had discussed SUDEP. However, 15 of them (94\%) said that they were glad their clinician had brought it up. Twenty-six families were interviewed postintervention, and 12 (46\%) reported having been told about SUDEP by their clinicians. The increase in reported clinician discussion rate of SUDEP from 16 of 75 to 12 of 26 was significant ( 25 percentage point difference, 95\% confidence interval: 1-49 percentage point increase, $p=0.03$ ). Of respondents who remembered, $80 \%$ reported that it was the clinician who initiated the conversation about SUDEP. Other measures were unaffected by the intervention. In a sensitivity analysis of recollection over time between visit and interview, we found the numbers of participants in the preintervention with visit-to-interview intervals of 101 to 200 days and 201 to 300 days are each comparable to the number in the postinterview interval of 0 to 100 days, yet the discussion rates were 27 to $28 \%$ in preintervention compared with $43 \%$ in postintervention. About $11 \%$ of the 27 respondents with visit-to-interview intervals $\geq 301$ days reported a SUDEP discussion.

\section{Discussion}

As in previous surveys, a small minority of families of children with epilepsy had heard about SUDEP from their clinicians. With the introduction of the CHICA-SUDEP module, the percentage of caregivers who reported discussing SUDEP with their child's clinician more than doubled from 21 to $46 \%$, and when the parent recalled who brought up the topic, $80 \%$ of the time it was the clinician. Our findings support CDS workflows that include patient-reported outcomes in chronic condition management.

The results are striking in that these were general pediatric clinics, not pediatric neurology subspecialty settings (CHICASUDEP was piloted in general pediatric clinics because the CHICA infrastructure already existed in these primary care clinics). Pediatric primary care providers have a low awareness of SUDEP and rate of discussion with patients. ${ }^{17}$ It is possible that neurologists and pediatric neurologists would respond even more to such an intervention, given their baseline fund of knowledge and experience with SUDEP. Indeed, quality improvement projects in epilepsy clinics in the United Kingdom introduced a SUDEP checklist and reported up to $80 \%$ of patients receiving a SUDEP assessment. Later, use of that checklist was associated with lower overall risk score and some lower specific risk factors. ${ }^{18,19}$ However, these studies are targeted to the adult population, whereas our work focused on pediatric patients and their families. Regardless, using automated screening, assessment, and decision support tools with longitudinal monitoring, such as those in our study, may have similar effect in the pediatric neurology domain.

Even with an increase in SUDEP discussion rate with our intervention, the majority (54\%) of caregivers still reported no SUDEP discussion. In comparison to other studies of physician prompting without a "hard stop" in EHRs (requiring clinicians to respond before proceeding), this was a successful intervention. Studies suggest that physicians respond to alerts in their EHRs $20 \%$ of the time or less. ${ }^{20,21}$ In 
Table 2 Results of the survey before and after introduction of the CHICA-SUDEP module (question-specific denominators noted, if applicable)

\begin{tabular}{|c|c|c|c|c|}
\hline Question & Response & $\begin{array}{l}\text { Before } \\
\text { intervention } \\
n(\%)\end{array}$ & $\begin{array}{l}\text { After } \\
\text { intervention } \\
n(\%)\end{array}$ & $p$-Value ${ }^{c}$ \\
\hline Number of Respondents & & 75 & 26 & \\
\hline \multirow[t]{2}{*}{ Did your doctor discuss SUDEP at the visit? } & Yes & $16(21.3)$ & $12(46.2)$ & 0.03 \\
\hline & No & $59(78.7)$ & $14(53.8)$ & \\
\hline \multirow{2}{*}{$\begin{array}{l}\text { Who brought up SUDEP at the visit? (Only asked post- } \\
\text { intervention to those who remembered, } n=10)^{\mathrm{a}}\end{array}$} & Provider & - & 8 out of $10(80.0)$ & \\
\hline & Parent & - & $2(20.0)$ & \\
\hline \multirow{2}{*}{$\begin{array}{l}\text { Were you glad your doctor brought up SUDEP? } \\
\text { (if doctor brought it up) }\end{array}$} & Yes & 15 of $16(93.8)$ & 7 of $7(100.0)^{b}$ & 1 \\
\hline & No & 1 of $16(6.2)$ & $0(0.0)$ & \\
\hline \multirow{3}{*}{$\begin{array}{l}\text { Do you feel you have a good enough understanding } \\
\text { about epilepsy that you are comfortable helping (name) } \\
\text { manage it? }\end{array}$} & Yes & $65(86.7)$ & $26(100.0)$ & 0.15 \\
\hline & No & $9(12.0)$ & $0(0.0)$ & \\
\hline & Don't know & $1(1.3)$ & $0(0.0)$ & \\
\hline \multirow{3}{*}{$\begin{array}{l}\text { Do you feel well educated about SUDEP? } \\
\text { (among those who had heard of SUDEP) }\end{array}$} & Yes & 17 of $21(81.0)$ & 6 of $10(60.0)$ & 0.46 \\
\hline & No & 1 of $21(4.8)$ & 1 of $10(10.0)$ & \\
\hline & $\begin{array}{l}\text { To a degree, } \\
\text { but I want more } \\
\text { information }\end{array}$ & 3 of $21(14.3)$ & 3 of $10(30.0)$ & \\
\hline
\end{tabular}

Abbreviations: CHICA, child health improvement through computer automation; SUDEP, sudden unexpected death in epilepsy.

'Two additional participants couldn't remember who brought up SUDEP.

bone missing value.

${ }^{c}$ Chi-square analysis.

general, satisfaction with CHICA is good, ${ }^{22}$ and our experience with other CHICA modules is that a response rate of around 50\% is typical. Our prior work suggests that lack of comfort with a topic is one barrier to responding to prompts and reminders. 23,24

An advantage of CHICA is that it will "remember" when this discussion hasn't happened (wasn't documented), and it will remind the clinician again at subsequent visits. Therefore, we expect the number of families who have the SUDEP discussion would likely go up over time. Additionally, we measured the caregiver's recollection of the discussion, which is a relatively distal yet important patient-centered outcome of alerting the provider, the provider discussing with the family, and the caregiver recalling the discussion. Even with a substantial percentage of clinicians not responding to the prompts, $47 \%$ of clinicians receiving a SUDEPrelated prompt reported having a SUDEP discussion. Our findings may underestimate the actual discussion rate that occurred between clinicians and families. ${ }^{25}$ Using patientcentered handouts can also increase patient-provider discussion rates and patient recall of medical information. ${ }^{25-27}$

We noted that $23 \%$ of the times that a family reported that their child had seizures or epilepsy, the clinician denied it. Parents sometimes describe nonseizure events as "seizures," or a clinician may determine that previous seizures did not constitute epilepsy. However, the initial screening question was developed as a sensitive way to assess for tonic-clonic seizures. Unfortunately, beyond caregiver's report, we had no way to confirm a diagnosis of epilepsy within the context of this pilot study.

A well-documented barrier to discussing SUDEP is concern about upsetting the patient or family. ${ }^{28,29}$ However, recent research indicates a risk-informed discussion of SUDEP does not have a negative effect on quality of life or mood, and may actually have a benefit for self-management in adult patients. ${ }^{30,31}$ One of our notable findings was that caregivers, both before and after the intervention, were glad their child's clinician had brought up SUDEP. This reinforces previous studies and policy statements strongly recommending discussion of SUDEP, preferably with written material, as parents and patients unequivocally expect. $3,28,32,33$

This pilot study is limited in some important ways. First, it is a before-after study design; a randomized trial would be stronger. Thus, secular trends, though not expected, could confound the results. Second, our strategy for recruiting patients differed slightly in the pre- and postintervention groups. Although this introduces possible bias, including the greater time between the visit and the phone interview, it seems unlikely that parents would forget a SUDEP discussion and it is hard to imagine it would more than double the rate of SUDEP discussions. Notably, there was a trend toward higher recollection inversely related to the time between 
visit and interview, largely driven by the extended time frames of $>300$ days. However, the predicted recollection for the postintervention period would still be well below what would be projected based on that trend. Telephone surveys suffer from low response rates. However, we were able to reach $66 \%$ of families attempted, better than other studies in underserved populations. ${ }^{34,35}$ The enrollment rate was higher in the preintervention period; we cannot explain this difference.

Our preintervention data query did not include codes for nonepileptic seizures, so patients with misclassified epilepsy may not have been included. We also acknowledge that our screening question assessing for "epilepsy or more than one seizure with stiffness and jerking" may not guarantee that the child has GTCs. However, the caregivers or patients reported here had to respond to that assessment at least twice in different occasions to reduce the chance of misclassifying their seizures. The screening question we developed is similar to the screening question for GTCs validated by Anand et $\mathrm{al}^{11}$ and others, ${ }^{12}$ which had $76 \%$ sensitivity and $99.5 \%$ specificity in a general population. Patient or caregiver questions and ICD diagnosis categories are both valid screening options for epilepsy. ${ }^{36}$ Furthermore, this system is intended to inform a learned intermediary (the physician), who presumably can differentiate absence seizures from GTCs, for example. Finally, this study was done in general pediatric clinics. Results in other settings, including neurologists' offices or other geographic locations, may be different.

About $52 \%$ of patients were considered to be at higher risk of SUDEP in our study because they had at least one seizure with stiffness and jerking in the preceding year. We found that $9 \%$ of patients also reported having trouble affording their antiseizure medication, 33\% reported not seeing their neurologist in the last year, and $12 \%$ said they had difficulty getting to their neurologist. The number of children at higher risk for SUDEP, combined with these barriers to decreasing their seizure frequency, point to the need for frank discussions with families about SUDEP and informatics-enhanced efforts to help them better manage their epilepsy. Fortunately, patients want this information and guidance. ${ }^{31,33}$

\section{Conclusion}

Future work should focus on the integration of computerbased decision support in the broader field of child neurology to help these patients and others facing chronic disease. Decision support systems can incorporate new risk factors that come to light. ${ }^{37}$ Our results happened in primary care clinics amidst all the other preventive care routinely happening; the subspecialist visit may be more accommodating to SUDEP discussions. Though limited by recall bias, our successful results contribute to the growing momentum of guideline-concordant subspecialty CDSS, extending beyond the typical preventive care reminders. The combination of patient and family input and prioritization of the child's needs offered by CHICA are a promising approach to achieving these goals.

\section{Clinical Relevance Statement}

Sudden unexpected death in epilepsy (SUDEP) is a rare but fatal risk. SUDEP is underdiscussed with at-risk patients and families. Computerized decision support doubled SUDEP counseling by general pediatricians and may help reduce SUDEP occurrence.

\section{Multiple Choice Questions}

1. Which of the following is a barrier to implementing computerized clinical decision support reminders in primary care?

a. Lack of recommendations for care.

b. Patients don't want to hear the content of the reminder.

c. Electronic health record systems often do not allow computerized decision support.

d. Clinicians not consistently using the automated system.

Correct Answer: The correct answer is option d. Clinicians have widely varying rates of responding to and interacting with automated decision support systems. Factors for this include interruptions at the wrong time in the workflow, lack of time, alert fatigue, and poorly worded prompts. Electronic health record systems often include functionality for decision support. There is often an abundance of recommendations for care, especially preventive health.

2. Which of the following is a likely reason a clinician may find the answer to a patient-facing screening question to be incorrect?

a. The patient does not know their medical history.

b. The screening question is formed to be highly sensitive.

c. An error in programming the screening question and answer report.

d. The patient intentionally gives a false answer.

Correct Answer: The correct answer is option b. A highly sensitive question will err toward identifying possible cases and have a corresponding risk of false positives. A common two-stage screening method is to use a highly sensitive question followed by a highly specific question. Thus a previsit questionnaire may want to catch all patients that might have a significant chance of the item under question, knowing that some may be falsely identified. The follow-up that occurs with other questions or even the clinician can rule out the false positives.

\section{Protection of Human and Animal Subjects}

The study was approved by the Indiana University Institutional Review Board.

\section{Funding}

This study was funded by Digital Health Solutions, LLC, through a grant provided by the Child Neurology Foundation. 


\section{Conflict of Interest}

R.W.G. has received grant support through Indiana University from Digital Health Solutions, LLC, and Pfizer, Inc. S.M.D. cofounded CHICA and Digital Health Solutions, LLC, which holds license from Indiana University to commercialize CHICA. T.J.S. and M.H. are employees of Greenwich Biosciences. J.B. has been reimbursed for consulting services by The Epilepsy Foundation, The Epilepsy Study Consortium, Eisai, UCB, Upsher-Smith, and Zogenix.

\section{Acknowledgments}

The authors gratefully acknowledge the Pediatric Research Network (PResNet) and Child Health Informatics Research and Development Laboratory (CHIRDL) at the Indiana University School of Medicine for their contributions and assistance.

\section{References}

1 Sveinsson O, Andersson T, Carlsson S, Tomson T. The incidence of SUDEP: a nationwide population-based cohort study. Neurology 2017;89(02):170-177

2 Keller AE, Whitney R, Li S-A, Pollanen MS, Donner EJ. Incidence of sudden unexpected death in epilepsy in children is similar to adults. Neurology 2018;91(02):e107-e111

3 Harden C, Tomson T, Gloss D, et al. Practice guideline summary: sudden unexpected death in epilepsy incidence rates and risk factors: Report of the Guideline Development, Dissemination, and Implementation Subcommittee of the American Academy of Neurology and the American Epilepsy Society. Neurology 2017; 88(17):1674-1680

4 Cooper MS, Mcintosh A, Crompton DE, et al. Mortality in Dravet syndrome. Epilepsy Res 2016;128:43-47

5 Anand V, Carroll AE, Biondich PG, Dugan TM, Downs SM. Pediatric decision support using adapted Arden Syntax. Artif Intell Med 2018;92:15-23

6 Anand V, Carroll AE, Downs SM. Automated primary care screening in pediatric waiting rooms. Pediatrics 2012;129(05): e1275-e1281

7 Hannon TS, Dugan TM, Saha CK, McKee SJ, Downs SM, Carroll AE. Effectiveness of computer automation for the diagnosis and management of childhood type 2 diabetes: a randomized clinical trial. JAMA Pediatr 2017;171(04):327-334

8 Zimet G, Dixon BE, Xiao S, et al. Simple and elaborated clinician reminder prompts for human papillomavirus vaccination: a randomized clinical trial. Acad Pediatr 2018;18(2S):S66-S71

9 Carroll AE, Bauer NS, Dugan TM, Anand V, Saha C, Downs SM. Use of a computerized decision aid for developmental surveillance and screening: a randomized clinical trial. JAMA Pediatr 2014;168 (09):815-821

10 Adlassnig K-P, Haug P, Jenders RA. Arden Syntax: then, now, and in the future. Artif Intell Med 2018;92:1-6

11 Anand K, Jain S, Paul E, Srivastava A, Sahariah SA, Kapoor SK. Development of a validated clinical case definition of generalized tonic-clonic seizures for use by community-based health care providers. Epilepsia 2005;46(05):743-750

12 Giuliano L, Cicero CE, Crespo Gómez EB, et al. A screening questionnaire for convulsive seizures: a three-stage field-validation in rural Bolivia. PLoS One 2017;12(03):e0173945

13 Modi AC, Monahan S, Daniels D, Glauser TA. Development and validation of the pediatric epilepsy medication self-management questionnaire. Epilepsy Behav 2010;18(1,2):94-99

14 Child Neurology Foundation. Sudden Unexpected Death in Epilepsy (SUDEP). Accessed May 28, 2019 at: https://www.childneurologyfoundation.org/SUDEP/
15 R Foundation for Statistical Computing. The R project for Statistical Computing. Accessed December 2, 2020 at: https://www.Rproject.org/

16 Harris PA, Taylor R, Thielke R, Payne J, Gonzalez N, Conde JG. Research electronic data capture (REDCap)-a metadata-driven methodology and workflow process for providing translational research informatics support. J Biomed Inform 2009;42(02): 377-381

17 Berl MM, Goodkin HP, Kroner BL, Bumbut A, Lapham G, Gaillard WD. Sudden death in epilepsy: knowledge among pediatric providers. J Pediatr 2017;188:291-293.e3

18 Shankar R, Newman C, Hanna J, et al. Keeping patients with epilepsy safe: a surmountable challenge? BMJ Qual Improv Rep 2015;4(01):u208167.w3252

19 Shankar R, Henley W, Boland C, et al. Decreasing the risk of sudden unexpected death in epilepsy: structured communication of risk factors for premature mortality in people with epilepsy. Eur J Neurol 2018;25(09):1121-1127

20 Shojania KG, Jennings A, Mayhew A, Ramsay C, Eccles M, Grimshaw J. Effect of point-of-care computer reminders on physician behaviour: a systematic review. CMAJ 2010;182(05):E216-E225

21 van der Sijs H, Aarts J, Vulto A, Berg M. Overriding of drug safety alerts in computerized physician order entry. J Am Med Inform Assoc 2006;13(02):138-147

22 Grout RW, Cheng ER, Carroll AE, Bauer NS, Downs SM. A six-year repeated evaluation of computerized clinical decision support system user acceptability. Int J Med Inform 2018;112:74-81

23 Downs SM, Anand V, Dugan TM, Carroll AE. You can lead a horse to water: physicians' responses to clinical reminders. AMIA Annu Symp Proc 2010;2010:167-171

24 Bauer NS, Carroll AE, Saha C, Downs SM. Experience with decision support system and comfort with topic predict clinicians' responses to alerts and reminders. J Am Med Inform Assoc 2016;23(e1):e125-e130

25 Watson PW, McKinstry B. A systematic review of interventions to improve recall of medical advice in healthcare consultations. J R Soc Med 2009;102(06):235-243

26 Barkin SL, Scheindlin B, Brown C, Ip E, Finch S, Wasserman RC. Anticipatory guidance topics: are more better? Ambul Pediatr 2005;5(06):372-376

27 Ancker JS, Send A, Hafeez B, Osorio SN, Abramson E. Health IT usability focus section: adapting EHR-Based medication instructions to comply with plain language guidance-A randomized experiment. Appl Clin Inform 2017;8(04):1127-1143

28 Gayatri NA, Morrall MCHJ, Jain V, Kashyape P, Pysden K, Ferrie C. Parental and physician beliefs regarding the provision and content of written sudden unexpected death in epilepsy (SUDEP) information. Epilepsia 2010;51(05):777-782

29 Friedman D, Donner EJ, Stephens D, Wright C, Devinsky O. Sudden unexpected death in epilepsy: knowledge and experience among U.S. and Canadian neurologists. Epilepsy Behav 2014; 35:13-18

30 Radhakrishnan DM, Ramanujam B, Srivastava P, Dash D, Tripathi M. Effect of providing sudden unexpected death in epilepsy (SUDEP) information to persons with epilepsy (PWE) and their caregivers-experience from a tertiary care hospital. Acta Neurol Scand 2018;138(05):417-424

31 Collard SS, Regmi P. Qualitative insights into the feelings, knowledge, and impact of SUDEP: a narrative synthesis. Epilepsy Behav 2019;94:20-28

32 Ramachandrannair R, Jack SM, Meaney BF, Ronen GM. SUDEP: what do parents want to know? Epilepsy Behav 2013;29(03): 560-564

33 Ramachandran Nair R, Jack SM, Strohm S. SUDEP: to discuss or not? Recommendations from bereaved relatives. Epilepsy Behav 2016;56:20-25

34 Sinclair M, O’Toole J, Malawaraarachchi M, Leder K. Comparison of response rates and cost-effectiveness for a community-based 
survey: postal, internet and telephone modes with generic or personalised recruitment approaches. BMC Med Res Methodol 2012;12(01):132

35 Knoll M, Soller L, Ben-Shoshan M, et al. The use of incentives in vulnerable populations for a telephone survey: a randomized controlled trial. BMC Res Notes 2012;5(01):572
36 Jetté N, Reid AY, Quan H, Hill MD, Wiebe S. How accurate is ICD coding for epilepsy? Epilepsia 2010;51(01):62-69

37 Sveinsson O, Andersson T, Mattsson P, Carlsson S, Tomson T. Clinical risk factors in SUDEP: a nationwide population-based case-control study. Neurology 2020;94(04):e419-e429 